\title{
Low-contrast letter charts in early diabetic retinopathy, ocular hypertension, glaucoma, and Parkinson's disease
}

\author{
D. REGAN AND D. NEIMA
}

From the Department of Ophthalmology, Dalhousie University, Halifax, Nova Scotia

SUmmary Diabetes can cause visual loss that is not detected by standard reading tests such as the Snellen test but can be detected by low-contrast letter charts. This visual loss is quite different from loss caused by refractive error. These low-contrast charts are diagnostically at least as sensitive as the sinewave grating contrast sensitivity test. They are inexpensive, and the test is brief and simple. Preliminary evidence is that patients with diabetes who have abnormal low-contrast chart results give abnormal intravenous fluorescein (IVF) test results, even though visual acuity is normal. Low-contrast charts also detect visual loss in patients with ocular hypertension, glaucoma, and Parkinson's disease, including patients with normal visual acuity.

The standard Snellen test requires a patient to read smaller and smaller letters. If the patient can read letters that subtend about $5 \mathrm{~min}$ arc, vision is conventionally supposed to be within the normal range. It has recently been found, however, that this assumption is not necessarily valid. The Snellen and similar reading tests are based on the assumption that spatial form vision is normal if the ability to see fine detail is unimpaired. For refractive disorders this assumption is indeed valid. But several diseases can degrade the ability to see objects of large or intermediate size while leaving the ability to see fine detail comparatively, or even completely, unaffected. These diseases include multiple sclerosis, ${ }^{1-3}$ glaucoma, and ocular hypertension..$^{4-8}$ In Parkinson's disease, however, although visual evoked potential delays have been found, ${ }^{9}$ contrast sensitivity losses have not been reported.

In the standard Snellen test the only variable is letter size. Consequently, a patient's ability to see objects of several different sizes cannot be tested with the standard Snellen chart. The reason that the standard chart tests a patient's ability to see the finest detail, rather than the ability to see letters of larger or intermediate size, is that the standard chart's letters are all of high contrast. In order to overcome this limitation we constructed a set of charts in which two

Correspondence to Dr D. Regan, Dalhousie University, Gerard Hall, 5303 Morris Street, Halifax, Nova Scotia, Canada B3J 1B6. quantities were varied, namely, letter contrast and letter size. ${ }^{10}$ The low-contrast chart tests the ability to see large letters, the intermediate contrast chart the ability to see letters of intermediate size, and the high contrast chart tests the ability to see small letters.

Low-contrast letter charts have several advantages compared with the grating test including cheapness and familiarity to the ophthalmologist. Compared with Arden gratings" the low-contrast letter charts have the advantage of providing the ophthalmologist with a check on the patient's criterion.

In a previous study on patients with multiple sclerosis we confirmed that this set of variablecontrast letter charts provides qualitatively similar diagnostic information to the sinewave grating test. ${ }^{10}$ In particular, the low-contrast letter charts detected visual dysfunction that was not detected by the standard high-contrast chart.

Early detection of visual dysfunction is important in diabetes. It has recently been found that sinewave grating tests can detect visual loss in this disease before Snellen acuity is affected. It occurred to us that, if low-contrast letter chart results paralleled these grating test results, the charts would offer a simple and convenient means of detecting subclinical visual loss. With this in mind we compared test results using low-contrast charts and sinewave gratings in patients with diabetes. We found that the low-contrast letter charts detected visual loss that was not detected by the standard Snellen test, and that the charts not 

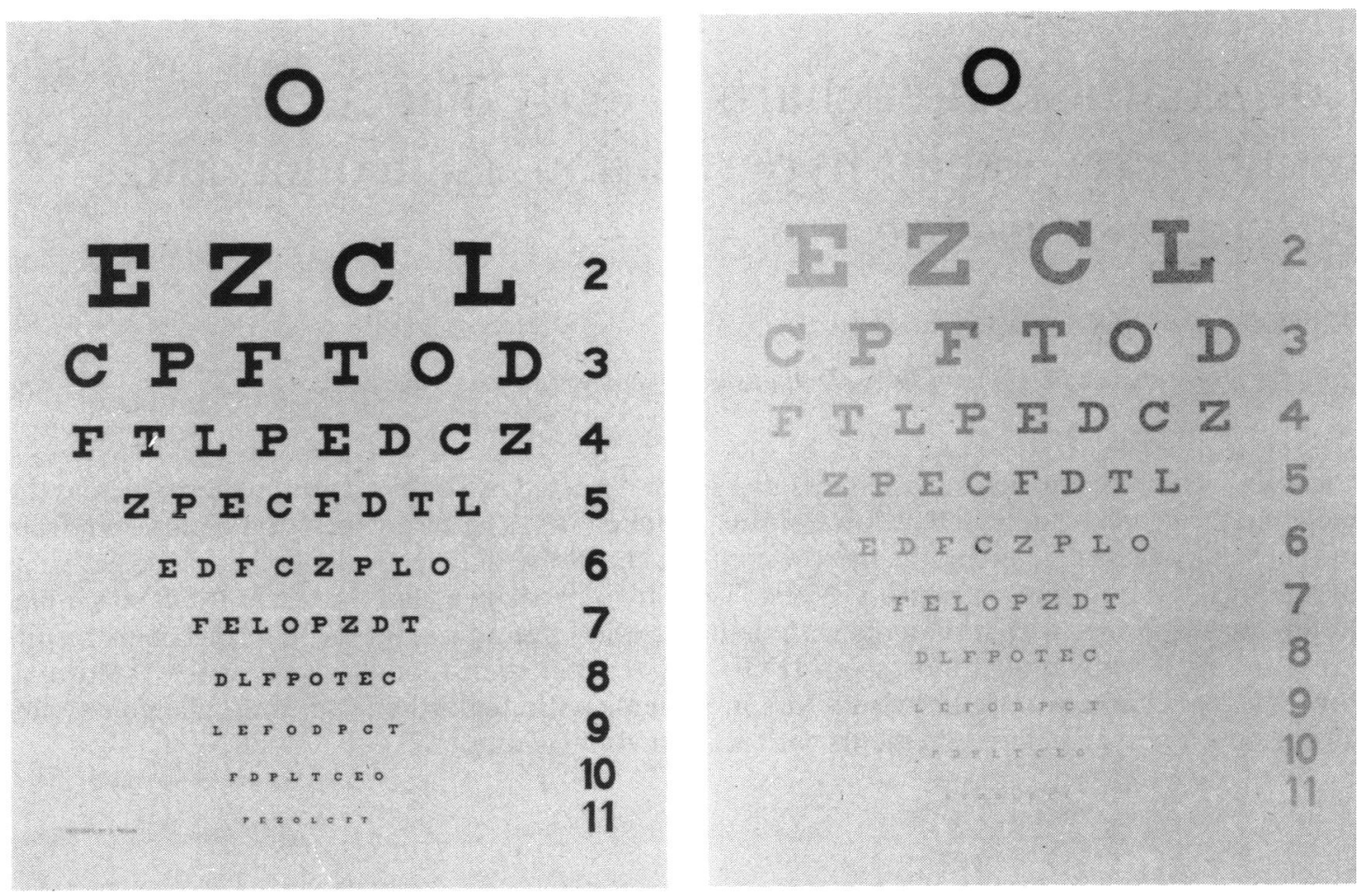

Fig. 1 Letter test slides showing high-contrast, intermediate-contrast, and low-contrast versions. 
only gave qualitatively similar results to the grating test but they were at least as sensitive. The lowcontrast letter test also detected visual loss in patients with ocular hypertension, glaucoma, and Parkinson's disease, even when Snellen acuity was normal.

\section{Methods}

APPARATUS

We tested subjects' vision using five specially prepared charts each of which measured $55 \times 30 \mathrm{~cm}$ and was painted on a white chart. One chart resembled the standard Snellen card. The letters were black. The contrast of this chart approximated $100 \%$ if we define letter contrast as $100\left(I_{2}-I_{1}\right) / I_{2}$, where $I_{2}$ is the luminance (that is, brightness) of the white card and $I_{1}$ is the luminance of the letter. The only difference between this high-contrast chart and the other four charts was that the letters were grey rather than black on the other charts, that is, the letters had contrasts below $100 \%$. The letters on any given chart had the same contrast. Three of the charts are illustrated in a previous article. " Contrasts for the five charts were as follows: $93 \%, 64 \%, 31 \%, 22 \%$, and $10 \%$. The charts were viewed from $600 \mathrm{~cm}$ and were illuminated by tungsten light so that the white parts of the cards had a luminance of $140 \mathrm{~cd} \mathrm{~m}^{-2}$ The low-contrast letter test has also been made as slides in a slightly different format from the charts. The slide format is shown in Fig. 1.

Vertical sinewave grating patterns were generated electronically and presented on a CRT. The grating occupied a circular area subtending $3.5^{\circ}$ at the eye, had a mean luminance of $20 \mathrm{~cd} \mathrm{~m}^{-2}$, and was viewed monocularly from $145 \mathrm{~cm}$. Spatial frequency was varied between 1 and 30 cycles deg $^{-1}$

\section{TEST PROCEDURE AND DATA ANALYSIS}

The patients read letters on a given chart and were required to guess at the letters they found hard to see. This requirement was important. We recorded the number of letters correctly read for each line. Fig. 2 presents these data in the following way. We noted the smallest letter size for which any letters at all were correctly read and repeated this for each of the five charts. We then plotted this smallest readable letter size as abscissa versus letter contrast as ordinate. We established normal limits by testing 20 correctly refracted control subjects (40 eyes) who had no history of ophthalmological disorder. We defined our normal limit for the letter test as two lines (2.5 SD) from the control mean. For the sinewave grating test we took our normal limit as $2.5 \mathrm{SD}$ from the control mean for contrast sensitivity.

The use of sinewave gratings in visual testing has been reviewed recently. ${ }^{12}$ We measured contrast

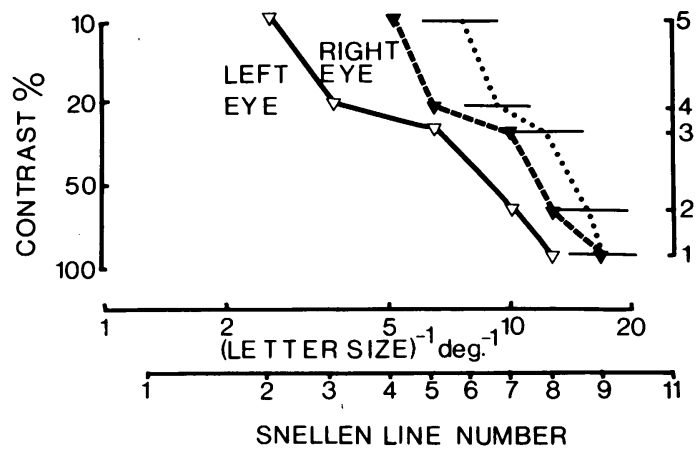

Fig. 2 Low-contrast letter chart results in a diabetic patient. Visual acuity was normal in both eyes for the standard highcontrast Snellen chart (No. 1), but the left eye was abnormal for low-contrast letter charts (Nos. 4 and 5). The dotted line is for control eyes, horizontal bars plotting \pm 1 standard deviation.

thresholds using a von Békésy tracking method as described previously ${ }^{13}$ testing up to seven spatial frequencies. Each measurement was repeated twice, except when the first two disagreed by more than 3 dB. A third repeat was then recorded.

\section{PATIENTS AND CONTROLS}

We tested 15 patients with diabetes (age range 24 to 75 years, mean 49 ), 12 patients with ocular hypertension (age range 47 to 71 years, mean 58 ), 10 patients with glaucoma (age range 41 to 73 years, mean 61), and 10 patients with Parkinson's disease (age range 41 to 79 years, mean 62 ). Control data were established with 20 subjects (age range 20 to 61 years, mean 37).

\section{Results}

Diabetes. In diagnostic sensitivity the low-contrast letter charts compare favourably with the sinewave grating test. Of 15 diabetic patients seven had visual loss on the grating test, and nine gave abnormal results on the low-contrast letter charts. Fig. 2 shows low-contrast letter test results for a diabetic patient. The dashed line is for the right eye and the continuous line for the left. Mean data for $\mathbf{4 0}$ control eyes are shown by the dotted line. The horizontal bars show \pm 1 standard deviation for the control group. Using the standard high-contrast Snellen chart (1 on righthand ordinate) visual acuity was within the normal range for both eyes. However, for the lowest-contrast chart (5 on right-hand ordinate) the left eye's performance was about 4 SD below the control mean. The left eye's defect was such that slightly greater contrast was required to see small letters than for a normal eye, but much greater contrast was required to see large letters than a normal eye required. The 
visual losses shown in Fig. 2 are quite different from those produced by refractive error. Blurring vision with lenses shifts the control curve bodily to the left, ${ }^{10}$ but the slope of the curve does not change, whereas the curve of the diabetic patient's left eye is clearly tilted.

In nine patients with visual acuities below 6/7.5 four had visual losses on the grating test. These losses were at low spatial frequencies and, therefore, demonstrated dysfunction that was due to visual pathway rather than refractive disorder.

Six patients had visual acuities of $6 / 7.5$ or better in both eyes. Independently of this study an IVF test was carried out in these patients as a routine diagnostic procedure. We compared the results of intravenous fluorescein tests (IVFs) of these six patients with the letter chart and grating test results. Three patients had abnormal letter and grating results. The IVFs of these three patients showed ischaemia of the parafoveal arcade and some degree of macular oedema. The three patients with normal IVFs had normal visual test results.

Ocular hypertension. Of 12 patients with ocular hypertension seven had low-contrast letter test abnormalities. Low-contrast letter charts detected visual loss in five of 10 patients who were normal on the standard Snellen test (visual acuity of $6 / 7.5$ or better).

Glaucoma. Of 10 patients with glaucoma four had low-contrast letter test abnormalities. One of these patients had normal Snellen acuity $(6 / 7 \cdot 5)$.

Parkinson's disease. Of 10 patients with Parkinson's disease five had low-contrast letter test abnormalities. Three of these patients had normal Snellen acuity $(6 / 7 \cdot 5$ or better).

\section{Discussion}

The finding that patients with multiple sclerosis can lose visual sensitivity to small objects, large objects, and objects of intermediate size somewhat independently has been explained by suggesting that the disease can involve neurons with large, small, or intermediate-sized receptive fields somewhat independently. ${ }^{12} 14$ Regan et al. ${ }^{15}$ reported evidence that cortical neurons are involved in multiple sclerosis, arguing from their finding that visual loss can be specific to the orientation of the test pattern and the fact that orientation-specific neurons are not found peripheral to primary visual cortex. Cortical pathophysiology is not, however, a likely explanation for the finding that glaucoma and ocular hypertension can degrade the ability to see large targets while sparing visual acuity. Regan and Neima ${ }^{s}$ suggested that the pathophysiology here is at the level of retinal ganglion cell dendrites, the most distal processes in the largest dendritic trees being the first to suffer functional loss. ${ }^{8}$

Our main finding is that, in diabetic patients, lowcontrast letter charts can detect visual loss that is not detected by the standard Snellen test, and that the low-contrast chart test is at least as sensitive to such visual loss as is the sinewave grating test. As a means of detecting such subclinical visual loss the lowcontrast letter charts test offers a practical office test with the advantages of cheapness, speed, an automatic check on the patient's response bias and familiarity of test procedure.

What is the pathophysiology responsible for our finding that the ability to see large letters can be degraded in diabetes, while visual acuity is comparatively spared? It is possible that the pathophysiology is similar to that suggested to occur in ocular hypertension, ${ }^{5}$ namely, that retinal ischaemia causes functional loss at the extremities of the largest dendritic trees of ganglion cells before smaller trees are affected. Some support for this suggestion is provided by the correlation between abnormal IVF records and abnormal letter chart results, though the number of patients is too small to support more than a tentative suggestion. Nevertheless, if this correlation is confirmed by an IVF study in progress then the low-contrast letter test would offer a simple non-invasive screening procedure for identifying the patients who would most benefit from IVF investigation.

We have been unable to find a previous report that, in Parkinson's disease, contrast sensitivity losses can occur that are not attributable to optical problems at the front of the eye. Our findings with ocular hypertension and glaucoma confirm that the low-contrast letter chart test is at least equal to the grating test as a means of detecting visual pathway dysfunction that is not detected by the standard Snellen test.

A limited number of low-contrast charts have been prepared in the form of $2 \times 2$ inch $(5 \times 5 \mathrm{~cm})$ slides as illustrated in Fig. 1. They can be obtained from author D.R. at no cost for purposes of ophthalmological research.

This research was supported by grants to D.R. from the National Eye Institute (EYO-3058) and from the Multiple Sclerosis Society of Canada.

\section{References}

1 Regan D, Silver R, Murray TJ. Visual acuity and contrast sensitivity in multiple sclerosis: hidden visual loss. Brain 1977; 100: 563-79.

2 Bodis-Wellner I, Hendley CD, Mylin LH, Thornton J. Visual evoked potentials and the visuogram in multiple sclerosis. Ann Neurol 1979; 5: 40-7.

3 Zimmern RL, Campbell FW, Wilkinson IMS. Subtle disturbances of vision after optic neuritis elicited by studying contrast sensitivity. J Neurol Neurosurg Psychiatry 1979; 42: 407-12.

4 Atkin A, Wolkstein M, Bodis-Wollner I, Anders M, Kels B, Podos SM. Interocular comparison of contrast sensitivities in 
glaucoma patients and subjects. Br J Ophthalmol 1980; 64: 858-62.

5 Regan D, Neima D. Balance between pattern and flicker sensitivities in the visual fields of ophthalmological patients. $\mathrm{Br} J$ Ophthalmol 1984; 68: 310-5.

6 Sokol S, Domar A, Moskowitz A. Utility of the Arden grating test in glaucoma screening: high false positive rate in normals over 50 years of age. Invest Ophthalmol Visual Sci 1980; 19: 1529-33.

7 Neima D, Regan D, LeBlanc R. Contrast perimetry and acuity perimetry in ocular hypertension and glaucoma. Invest Ophthalmol Visual Sci 1983; 24 (suppl): 103.

8 Neima D, LeBlanc R, Regan D. Visual field defects in ocular hypertension and glaucoma. Arch Ophthalmol 1984; 102: 1042-5.

9 Bodis-Wollner I, Yahr MD. Measurements of visual evoked potentials in Parkinson's discase. Brain 1978; 101: 661-71.
10 Regan D, Neima D. Low-contrast letter charts as a test of visual function. Ophthalmology 1983; 90: 1192-200.

11 Arden GB, Jacobson JJ. A simple grating test for contrast sensitivity: preliminary results indicate value in screening for glaucoma. Invest Ophthalmol Visual Sci 1978; 17: 23-32.

12 Wolkstein M, Atkin A, Bodis-Wollner I. Contrast sensitivity in retinal disease. Ophthalmology 1980; 87: 1140-9.

13 Regan D, Beverley KI. Visual fields described by contrast sensitivity, by acuity, and by relative sensitivity to different orientations. Invest Ophthalmol Visual Sci 1983; 24: 754-9.

14 Bodis-Wollner I. Vulnerability of spatial frequency channels in cerebral lesions. Nature 1976; 261: 309-11.

15 Regan D, Whitlock J, Murray TJ, Beverley JI. Orientationspecific losses of contrast sensitivity in multiple sclerosis. Invest Ophthalmol Visual Sci 1980; 19: 324-8. 